\title{
GENERALIZED DOUBLE INTEGRAL INVOLVING KAMPÉ DE FÉRIET FUNCTION
}

\author{
Yong Sup Kim, Shoukat Ali, and Navratna Rathie
}

\begin{abstract}
The aim of this paper is to obtain twenty five Eulerian type double integrals in the form of a general double integral involving Kampé de Fériet function. The results are derived with the help of the generalized classical Watson's theorem obtained earlier by Lavoie, Grondin and Rathie. A few interesting special cases of our main result are also given.
\end{abstract}

\section{Introduction}

We recall the definition of generalized Kampé de Fériet function as follows [12]

$$
\begin{aligned}
& F_{l: m ; n}^{p: q ; k}\left[\begin{array}{llll}
\left(a_{p}\right) & :\left(b_{q}\right) & ;\left(c_{k}\right) & ; \\
\left(\alpha_{l}\right) & :\left(\beta_{m}\right) & ;\left(\gamma_{n}\right) & ;
\end{array}\right] \\
& =\sum_{r, s=0}^{\infty} \frac{\prod_{j=1}^{p}\left(a_{j}\right)_{r+s} \prod_{j=1}^{q}\left(b_{j}\right)_{r} \prod_{j=1}^{k}\left(c_{j}\right)_{s} x^{r} y^{s}}{\prod_{j=1}^{l}\left(\alpha_{j}\right)_{r+s} \prod_{j=1}^{m}\left(\beta_{j}\right)_{r} \prod_{j=1}^{n}\left(\gamma_{j}\right)_{s} r ! s !},
\end{aligned}
$$

where, for convergence,

(i) $p+q<l+m+1, p+k<l+n+1,|x|<\infty,|y|<\infty$ or

(ii) $p+q<l+m+1, p+k<l+n+1$ and

$$
\begin{cases}|x|^{\frac{1}{p-l}}+|y|^{\frac{1}{p-l}}<1 & \text {, if } p>l \\ \max \{|\mathrm{x}|,|\mathrm{y}|\}<1 & \text {, if } p \leq l .\end{cases}
$$

Although the double hypergeometric sreies defined by (1.1) reduces to the Kampé de Fériet function [2] in the special case: $q=k$ and $m=n$,

yet it is usually refereed to in the literature as the Kampé de Fériet

Received January 18, 2011. Accepted February 17, 2011.

2000 Mathematics Subject Classification. 33C20, 33C65, 33C70

Key words and phrases. Kampé de Fériet function, Watson's theorem, double integral 
series. The Kampé de Fériet function defined in (1.1) can be specialized to be expressed in terms of generalized hypergeometric series, among other things, as following instances:

$$
\begin{aligned}
& F_{q: 0 ; 0}^{p: 0 ; 0}\left[\begin{array}{l}
\alpha_{1}, \cdots, \alpha_{p} ; \\
\beta_{1}, \cdots, \beta_{q} ;
\end{array} \quad x, y\right]={ }_{p} F_{q}\left[\begin{array}{l}
\alpha_{1}, \cdots, \alpha_{p} \\
\beta_{1}, \cdots, \beta_{q}
\end{array} ; x+y\right] \\
& F_{0: q ; s}^{0: p ; r}\left[\begin{array}{l}
-; \alpha_{1}, \cdots, \alpha_{p} ; \gamma_{1}, \cdots, \gamma_{r} \\
-; \beta_{1}, \cdots, \beta_{q} ; \delta_{1}, \cdots, \delta_{s}
\end{array} \quad x, y\right] \\
& ={ }_{p} F_{q}\left[\begin{array}{l}
\alpha_{1}, \cdots, \alpha_{p} \\
\beta_{1}, \cdots, \beta_{q}
\end{array} ; x\right]{ }_{r} F_{s}\left[\begin{array}{l}
\gamma_{1}, \cdots, \gamma_{r} \\
\delta_{1}, \cdots, \delta_{s}
\end{array} ; y\right] \text {. }
\end{aligned}
$$

$$
\begin{gathered}
F_{q: 0 ; 0}^{p: 1 ; 1}\left[\begin{array}{l}
\alpha_{1}, \cdots, \alpha_{p} ; \nu ; \sigma \\
\beta_{1}, \cdots, \beta_{q} ;-;-
\end{array}, x\right]={ }_{p+1} F_{q}\left[\begin{array}{l}
\alpha_{1}, \cdots, \alpha_{p} ; \nu+\sigma \\
\beta_{1}, \cdots, \beta_{q}
\end{array} ; x\right] . \\
\left.F_{q: 1 ; 1}^{p: 0 ; 0}\left[\begin{array}{l}
\alpha_{1}, \cdots, \alpha_{p} ;-;- \\
\beta_{1}, \cdots, \beta_{q} ; \nu ; \sigma
\end{array}\right] x, x\right] \\
={ }_{p+2} F_{q+3}\left[\begin{array}{l}
\alpha_{1}, \cdots, \alpha_{p} ; \Delta(2 ; \nu+\sigma-1) \\
\beta_{1}, \cdots, \beta_{q}, \nu, \sigma, \nu+\sigma-1
\end{array} ; 4 x\right],
\end{gathered}
$$

where, and in what follows, $\Delta(l ; \lambda)$ abbreviates the array of $l$ parameters

$$
\frac{\lambda}{l}, \frac{(\lambda+1)}{l}, \cdots, \frac{(\lambda+l-1)}{l}, l=1,2,3, \ldots
$$

For more details, see Srivastava and Karlsson [12, pp. 28 - 32].

\section{Results required}

The following results will be required in our present investigations.

$$
\begin{aligned}
& \int_{0}^{1} \int_{0}^{1} y^{c}(1-x)^{c-1}(1-y)^{c+j-1}(1-x y)^{1-2 c} \\
& \times_{2} F_{1}\left[\begin{array}{l}
a, b \\
\frac{1}{2}(a+b+1+i)
\end{array} ; \frac{y(1-x)}{(1-x y)}\right] d x d y \\
& \left.=\frac{\Gamma(c) \Gamma(c+j)}{\Gamma(2 c+j)} 3 F_{2}\left[\begin{array}{l}
a, b, c \\
\frac{1}{2}(a+b+1+i), 2 c+j
\end{array}\right] 1\right]
\end{aligned}
$$


provided $\Re(c)>0, \Re(c+j)>0$ and $\Re(2 c-a-b+i+1)>0$ for $i, j=0, \pm 1, \pm 2$.

This result (2.1) is a special case of a general double integral given in Edward [4].

In 1992, Lavoie et al. [8] have given the generalization of the Watson's theorem on the sum of a ${ }_{3} F_{2}$ and obtained the following twenty five results in the form of a single result:

$$
\begin{aligned}
& { }_{3} F_{2}\left(\begin{array}{l}
a, \quad b, \\
a+b+1+i, 2 c+j
\end{array} \quad ; 1\right) \\
= & A_{i, j} \frac{2^{a+b+i-2} \Gamma\left(\frac{1}{2}(a+b+i+1)\right) \Gamma\left(c+\left[\frac{1}{2} j\right]+\frac{1}{2}\right) \Gamma\left(c-\frac{1}{2}(a+b+|i+j|-j-1)\right)}{\Gamma\left(\frac{1}{2}\right) \Gamma(a) \Gamma(b)} \\
& \times\left\{B_{i, j} \frac{\Gamma\left(\frac{1}{2} a+\frac{1}{4}\left(1-(-1)^{i}\right)\right) \Gamma\left(\frac{1}{2} b\right)}{\Gamma\left(c-\frac{1}{2} a+\frac{1}{2}+\left[\frac{1}{2} j\right]-\frac{1}{4}(-1)^{j}\left(1-(-1)^{i}\right)\right) \Gamma\left(c-\frac{1}{2} b+\frac{1}{2}+\left[\frac{1}{2} j\right]\right)}\right. \\
& \left.+C_{i, j} \frac{\Gamma\left(\frac{1}{2} a+\frac{1}{4}\left(1-(-1)^{i}\right)\right) \Gamma\left(\frac{1}{2} b+\frac{1}{2}\right)}{\Gamma\left(c-\frac{1}{2} a+\left[\frac{1}{2}(j+1)\right]+\frac{1}{4}(-1)^{j}\left(1-(-1)^{i}\right)\right) \Gamma\left(c-\frac{1}{2} b+\left[\frac{1}{2}(j+1)\right]\right)}\right\}
\end{aligned}
$$

provided $\Re(2 c-a-b)>-1-i-2 j$ with $i, j=0, \pm 1, \pm 2$. Here and in what follows, $[x]$ is the greatest integer less than or equal to $x$ and $|x|$ denotes the usual absolute value of $x$. The coefficients $A_{i, j}, B_{i, j}$ and $C_{i, j}$ are given respectively in [10].

\section{Main results}

The following results for reducibility of Kampé de Fériet function will be established in this section.

$$
\begin{aligned}
& \int_{0}^{1} \int_{0}^{1} y^{c}(1-x)^{c-1}(1-y)^{c+j-1}(1-x y)^{1-2 c}{ }_{2} F_{1}\left[\begin{array}{l}
a, b \\
\frac{1}{2}(a+b+1+i) ; \frac{y(1-x)}{(1-x y)}
\end{array}\right] \\
& \times F_{\nu ; \rho: \rho}^{\lambda ; \mu: \mu}\left[\begin{array}{lllll}
\left(\alpha_{\lambda}\right) & :\left(\beta_{\mu}\right) & ;\left(\beta_{\mu}^{\prime}\right) & ; & z_{1} y(1-x)(1-y)(1-x y)^{-2} \\
\left(\gamma_{\nu}\right) & :\left(\delta_{\rho}\right) & ;\left(\delta_{\rho}^{\prime}\right) & ; & z_{2} y(1-x)(1-y)(1-x y)^{-2}
\end{array}\right] d x d y
\end{aligned}
$$




$$
\begin{aligned}
& =\sum_{p, q=0}^{\infty} A_{p, q} z_{1}{ }^{p} z_{2}{ }^{q} \frac{\Gamma(c+p+q) \Gamma(c+p+q+j)}{\Gamma(2 c+2 p+2 q+j)} \\
& A_{i, j} \frac{\left.2^{a+b+i-2} \Gamma\left(\frac{1}{2}(a+b+i+1)\right)\right) \Gamma\left(c+p+q+\frac{1}{2}[j]+\frac{1}{2}\right) \Gamma\left(c+p+q-\frac{1}{2}(a+b+|i+j|-j-1)\right)}{\Gamma\left(\frac{1}{2}\right) \Gamma(a) \Gamma(b)} \\
& \times\left\{B_{i, j} \frac{\Gamma\left(\frac{1}{2} a+\frac{1}{4}\left(1-(-1)^{i}\right)\right) \Gamma\left(\frac{1}{2} b\right)}{\Gamma\left(c+p+q-\frac{1}{2} a+\frac{1}{2}+\left[\frac{1}{2} j\right]-\frac{1}{4}(-1)^{j}\left(1-(-1)^{i}\right)\right) \Gamma\left(c+p+q-\frac{1}{2} b+\frac{1}{2}+\left[\frac{1}{2} j\right]\right)}\right. \\
& \left.+C_{i, j} \frac{\Gamma\left(\frac{1}{2} a+\frac{1}{4}\left(1-(-1)^{i}\right)\right) \Gamma\left(\frac{1}{2} b+\frac{1}{2}\right)}{\Gamma\left(c+p+q-\frac{1}{2} a+\frac{1}{2}+\left[\frac{1}{2}(j+1)\right]-\frac{1}{4}(-1)^{j}\left(1-(-1)^{i}\right)\right) \Gamma\left(c+p+q-\frac{1}{2} b+\left[\frac{1}{2}(j+1)\right]\right)}\right\}
\end{aligned}
$$

provided $\Re(c)>0$, for $j=-1,-2 ; \Re(c+j)>0$ for $j=0,1,2$. Also, (i) $\lambda+\mu<\nu+\rho+1,\left|z_{1}\right|<\infty,\left|z_{2}\right|<\infty$ or

(ii) $\lambda+\mu=\nu+\rho+1$ and

$$
\begin{cases}\left|z_{1}\right|^{\frac{1}{\lambda-\nu}}+\left|z_{2}\right|^{\frac{1}{\lambda-\nu}}<1 & \text {, if } \lambda>\nu \\ \max \left\{\left|z_{1}\right|,\left|z_{2}\right|\right\}<1 & \text {, if } \lambda \leq \nu\end{cases}
$$

Also, the coefficients $A_{i, j}, B_{i, j}$ and $C_{i, j}$ can obtain from the tables given in [10] by replacing $c$ by $c+p+q$,

$$
A_{p, q}=\frac{\prod_{j=1}^{\lambda}\left(\alpha_{j}\right)_{p+q} \prod_{j=1}^{\mu}\left(\beta_{j}\right)_{p} \prod_{j=1}^{\mu}\left(\beta_{j}^{\prime}\right)_{q}}{\prod_{j=1}^{\nu}\left(\gamma_{j}\right)_{p+q} \prod_{j=1}^{\rho}\left(\delta_{j}\right)_{p} \prod_{j=1}^{\rho}\left(\delta_{j}^{\prime}\right)_{q} p ! q !} .
$$

\section{Proof of (3.1)}

To prove (3.1), we proceed as follows : Let

$$
\begin{aligned}
I= & \int_{0}^{1} \int_{0}^{1} y^{c}(1-x)^{c-1}(1-y)^{c+j-1}(1-x y)^{1-2 c}{ }_{2} F_{1}\left[\begin{array}{ll}
a, b \\
\frac{1}{2}(a+b+1+i)
\end{array} ; \frac{y(1-x)}{(1-x y)}\right] \\
& \times F_{\nu ; \rho: \rho}^{\lambda ; \mu}\left[\begin{array}{lllll}
\left(\alpha_{\lambda}\right) & :\left(\beta_{\mu}\right) & ;\left(\beta_{\mu}^{\prime}\right) & ; & z_{1} y(1-x)(1-y)(1-x y)^{-2} \\
\left(\gamma_{\nu}\right) & :\left(\delta_{\rho}\right) & ;\left(\delta_{\rho}^{\prime}\right) & ; & z_{2} y(1-x)(1-y)(1-x y)^{-2}
\end{array}\right] d x d y .
\end{aligned}
$$

Expressing Kampé de Fériet function as a double series, we have (4.2)

$$
\begin{aligned}
I= & \int_{0}^{1} \int_{0}^{1} y^{c}(1-x)^{c-1}(1-y)^{c+j-1}(1-x y)^{1-2 c}{ }_{2} F_{1}\left[\begin{array}{l}
a, b \\
\frac{1}{2}(a+b+1+i)
\end{array} ; \frac{y(1-x)}{(1-x y)}\right] \\
& \cdot \sum_{p, q=0}^{\infty} A_{p, q} z_{1}{ }^{p} z_{2}{ }^{q} y^{p}(1-x)^{p}(1-y)^{p}(1-x y)^{-2 p} y^{q}(1-x)^{q}(1-y)^{q}(1-x y)^{-2 q} d x d y,
\end{aligned}
$$


where

$$
A_{p, q}=\frac{\prod_{j=1}^{\lambda}\left(\alpha_{j}\right)_{p+q} \prod_{j=1}^{\mu}\left(\beta_{j}\right)_{p} \prod_{j=1}^{\mu}\left(\beta_{j}^{\prime}\right)_{q}}{\prod_{j=1}^{\nu}\left(\gamma_{j}\right)_{p+q} \prod_{j=1}^{\rho}\left(\delta_{j}\right)_{p} \prod_{j=1}^{\rho}\left(\delta_{j}^{\prime}\right)_{q} p ! q !} .
$$

Changing the order of integration and summatiom which is justified due to the uniformly convergence of the series, we derive

$$
\begin{aligned}
& I=\sum_{p, q=0}^{\infty} A_{p, q} z_{1}{ }^{p} z_{2}{ }^{q} \int_{0}^{1} \int_{0}^{1} y^{c+p+q}(1-x)^{c+p+q-1}(1-y)^{c+p+q+j-1} \\
& (1-x y)^{1-2(c+p+q)} \times{ }_{2} F_{1}\left[\begin{array}{l}
a, b \\
\frac{1}{2}(a+b+1+i)
\end{array} ; \frac{y(1-x)}{(1-x y)}\right] d x d y
\end{aligned}
$$

which, upon using (2.1), becomes

$$
\begin{aligned}
& I=\sum_{p, q=0}^{\infty} A_{p, q} z_{1}{ }^{p} z_{2} q \frac{\Gamma(c+p+q) \Gamma(c+p+q+j)}{\Gamma(2 c+2 p+2 q+j)} \\
& \left.\times{ }_{3} F_{2}\left[\begin{array}{l}
a, b, c+p+q \\
\frac{1}{2}(a+b+1+i), 2(c+p+q)+j
\end{array}\right] 1\right] .
\end{aligned}
$$

By making use of (2.2) and replacing $c$ by $c+p+q$, we finally arrive at the right-hand side of (3.1). This completes the proof of (3.1).

\section{Special cases}

In this section, we shall mention some of the interesting special cases of main result (3.1). 
(i) In (3.1), if we take $i=j=0$, then, we have, after a little simplification, the following transformation formula:

$$
\begin{aligned}
& \int_{0}^{1} \int_{0}^{1} y^{c}(1-x)^{c-1}(1-y)^{c-1}(1-x y)^{1-2 c}{ }_{2} F_{1}\left[\begin{array}{l}
a, b \\
\frac{1}{2}(a+b+1) ; \frac{y(1-x)}{(1-x y)}
\end{array}\right] \\
& \times F_{\nu ; \rho: \rho}^{\lambda ; \mu: \mu}\left[\begin{array}{lllll}
\left(\alpha_{\lambda}\right) & :\left(\beta_{\mu}\right) & ;\left(\beta_{\mu}^{\prime}\right) & ; & z_{1} y(1-x)(1-y)(1-x y)^{-2} \\
\left(\gamma_{\nu}\right) & :\left(\delta_{\rho}\right) & ;\left(\delta_{\rho}^{\prime}\right) & ; & z_{2} y(1-x)(1-y)(1-x y)^{-2}
\end{array}\right] d x d y \\
& =\frac{2^{a+b+2 c-1} \Gamma\left(\frac{1}{2}(a+b+1)\right) \Gamma\left(\frac{1}{2} a\right) \Gamma\left(\frac{1}{2} b\right) \Gamma(c) \Gamma\left(c-\frac{1}{2} a-\frac{1}{2} b+\frac{1}{2}\right)}{\Gamma(a) \Gamma(b) \Gamma\left(c-\frac{1}{2} a+\frac{1}{2}\right) \Gamma\left(c-\frac{1}{2} b+\frac{1}{2}\right)} \\
& \times F_{\nu+2 ; \rho: \rho}^{\lambda+2 ; \mu: \mu}\left[\begin{array}{llll}
\left(\alpha_{\lambda}\right), c, c+\frac{1}{2}(1-a-b) & :\left(\beta_{\mu}\right) & ;\left(\beta_{\mu}^{\prime}\right) & ; \\
\left(\gamma_{\nu}\right), c+\frac{z_{1}}{2}(1-a), c+\frac{1}{2}(1-b) & :\left(\delta_{\rho}\right) & ;\left(\delta_{\rho}^{\prime}\right) & ;
\end{array}\right]
\end{aligned}
$$

provided that the conditions easily obtainable from (3.1) are satisfied.

(ii) In (3.1), if we take $i=0, j=-1$, then, we have, after a little simplification, the following transformation formula:

$$
\begin{aligned}
& \int_{0}^{1} \int_{0}^{1} y^{c}(1-x)^{c-1}(1-y)^{c-2}(1-x y)^{1-2 c}{ }_{2} F_{1}\left[\begin{array}{l}
a, b \\
\frac{1}{2}(a+b+1) ; \frac{y(1-x)}{(1-x y)}
\end{array}\right] \\
& \times F_{\nu ; \rho: \rho}^{\lambda ; \mu: \mu}\left[\begin{array}{lllll}
\left(\alpha_{\lambda}\right) & :\left(\beta_{\mu}\right) & ;\left(\beta_{\mu}^{\prime}\right) & ; & z_{1} y(1-x)(1-y)(1-x y)^{-2} \\
\left(\gamma_{\nu}\right) & :\left(\delta_{\rho}\right) & ;\left(\delta_{\rho}^{\prime}\right) & ; & z_{2} y(1-x)(1-y)(1-x y)^{-2}
\end{array}\right] d x d y \\
& =\frac{2^{a+b+2 c} \Gamma\left(\frac{1}{2}(a+b+1)\right) \Gamma\left(\frac{1}{2} a\right) \Gamma\left(\frac{1}{2} b\right) \Gamma(c-1) \Gamma\left(c-\frac{1}{2} a-\frac{1}{2} b-\frac{1}{2}\right)}{\Gamma(a) \Gamma(b) \Gamma\left(c-\frac{1}{2} a-\frac{1}{2}\right) \Gamma\left(c-\frac{1}{2} b-\frac{1}{2}\right)}
\end{aligned}
$$

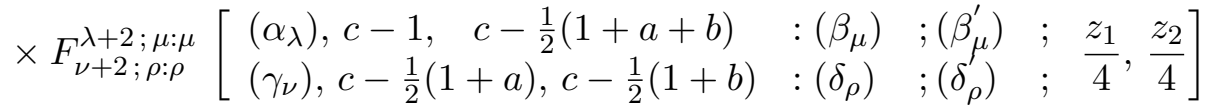

$$
\begin{aligned}
& +\frac{2^{a+b-2 c} \Gamma\left(\frac{1}{2}(a+b+1)\right) \Gamma\left(\frac{1}{2} a+\frac{1}{2}\right) \Gamma\left(\frac{1}{2} b+\frac{1}{2}\right) \Gamma(c-1) \Gamma\left(c-\frac{1}{2} a-\frac{1}{2} b-\frac{1}{2}\right)}{\Gamma(a) \Gamma(b) \Gamma\left(c-\frac{1}{2} a\right) \Gamma\left(c-\frac{1}{2} b\right)}
\end{aligned}
$$

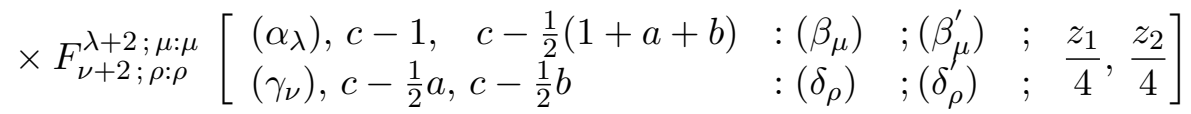

provided that the conditions easily obtainable from (3.1) are satisfied. 
(iii) In (3.1), if we take $i=0, j=1$, then, we have, after a little simplification, the following transformation formula:

$$
\begin{aligned}
& \int_{0}^{1} \int_{0}^{1} y^{c}(1-x)^{c-1}(1-y)^{c}(1-x y)^{1-2 c}{ }_{2} F_{1}\left[\begin{array}{l}
a, b \\
\frac{1}{2}(a+b+1) ; \frac{y(1-x)}{(1-x y)}
\end{array}\right] \\
& \times F_{\nu ; \rho: \rho}^{\lambda ; \mu: \mu}\left[\begin{array}{lllll}
\left(\alpha_{\lambda}\right) & :\left(\beta_{\mu}\right) & ;\left(\beta_{\mu}^{\prime}\right) & ; & z_{1} y(1-x)(1-y)(1-x y)^{-2} \\
\left(\gamma_{\nu}\right) & :\left(\delta_{\rho}\right) & ;\left(\delta_{\rho}^{\prime}\right) & ; & z_{2} y(1-x)(1-y)(1-x y)^{-2}
\end{array}\right] d x d y \\
& =\frac{2^{a+b-2 c-2} \Gamma\left(\frac{1}{2}(a+b+1)\right) \Gamma\left(\frac{1}{2} a\right) \Gamma\left(\frac{1}{2} b\right) \Gamma(c) \Gamma\left(c-\frac{1}{2} a-\frac{1}{2} b+\frac{1}{2}\right)}{\Gamma(a) \Gamma(b) \Gamma\left(c-\frac{1}{2} a+\frac{1}{2}\right) \Gamma\left(c-\frac{1}{2} b+\frac{1}{2}\right)} \\
& \times F_{\nu+2 ; \rho: \rho}^{\lambda+2 ; \mu: \mu}\left[\begin{array}{llll}
\left(\alpha_{\lambda}\right), c, c+\frac{1}{2}(1-a-b) & :\left(\beta_{\mu}\right) & ;\left(\beta_{\mu}^{\prime}\right) & ; \\
\left(\gamma_{\nu}\right), c+\frac{z_{1}}{2}(1-a), c+\frac{1}{2}(1-b) & :\left(\delta_{\rho}\right) & ;\left(\delta_{\rho}^{\prime}\right) & ;
\end{array}\right] \\
& -\frac{2^{a+b-2 c-2} \Gamma\left(\frac{1}{2}(a+b+1)\right) \Gamma\left(\frac{1}{2} a+\frac{1}{2}\right) \Gamma\left(\frac{1}{2} b+\frac{1}{2}\right) \Gamma(c) \Gamma\left(c-\frac{1}{2} a-\frac{1}{2} b+\frac{1}{2}\right)}{\Gamma(a) \Gamma(b) \Gamma\left(c-\frac{1}{2} a+1\right) \Gamma\left(c-\frac{1}{2} b+1\right)} \\
& \times F_{\nu+2 ; \rho: \rho}^{\lambda+2 ; \mu: \mu}\left[\begin{array}{lllll}
\left(\alpha_{\lambda}\right), c, c+\frac{1}{2}(1-a-b) & :\left(\beta_{\mu}\right) & ;\left(\beta_{\mu}^{\prime}\right) & ; & z_{1} \\
\left(\gamma_{\nu}\right), c-\frac{z_{2}}{2} a+1, c-\frac{1}{2} b+1 & :\left(\delta_{\rho}\right) & ;\left(\delta_{\rho}^{\prime}\right) & ; & 4
\end{array}\right]
\end{aligned}
$$

provided that the conditions easily obtainable from (3.1) are satisfied. Similarly other results can also obtain.

\section{References}

[1] C. Adiga and T. Kim, On a generalization of Sándor's function, Proc. Jangjeon Math. Soc.5(2)(2002), 119-129.

[2] P. Appell et J. Kampé de Fériet, Fonctions Hypergéométriques et Hypersphériques Polynomes d'Hermite, Gauthier-Villars, Paris, 1926.

[3] J. Choi, A. K. Rathie, and H. Harsh, Remarks on a summation formula for three variables hypergeometric function $X_{8}$ and certain hypergeometric transformations, East Asian Math. J. 25(4)(2009), 481-486.

[4] J. Edward, A treatise on the integral calculus, Vol. II, Chelsea Publishing Company, New York,1922.

[5] H. Exton, hypergeometric functions of three variables, J. Indian acad. Math. 4(1982), 113-119.

[6] T. Kim, M. S. M. Naika, S. C. Kumar, L. C. Jang, Y. H. Kim and B. Lee, On some new Schläfli-type cubic modular equations, Adv. Stud. Contemp. Math. 20(1)(2010), 63-80.

[7] Y. S. Kim, J. Choi, and A. K. Rathie, Remark on two results by Padmanabham for Exton's triple hypergeometric series $X_{8}$, Honam Math. J. 27(4)(2005), 603608. 
[8] Y. S. Kim and A. K. Rathie, On an extension formula for the triple hypergeometric series $X_{8}$ due to Exton, Bull. Korean Math. Soc. 44(4)(2007), 743-751.

[9] Y. S. Kim and A. K. Rathie, Another method for Padmanabham's transformation formula for Exton's triple hypergeometric series $X_{8}$ for On an extension formula for the triple hypergeometric series $X_{8}$, Commun. Korean Math. Soc. 24(4)(2009), 517-521.

[10] J. L. Lavoie, F. Grondin and A. K. Rathie Generalizations of Watson's theorem on the sum of a ${ }_{3} F_{2}$, Indian J. Math., 32(1)(1992), 23-32.

[11] S. W. Lee and Y. S. Kim, An extension of the triple hypergeometric series by Exton, Honam Math. J. 32(1)(2010), 61-71.

[12] H. M. Srivastava and P. W. Karlsson(1985), Multiple Gaussian Hypergeometric Series, Halsted Press (Ellis Horwood Limited, Chichester); Wiley, New York, Chichester, Brisbane, and Toronto.

[13] Z. Zhang and Y. Zhang, Summation formulas of $q$-series by modified Abel's lemma, Adv. Stud. Contemp. Math.17(2)(2008), 119-129.

Yong Sup Kim

Department of Mathematics Education, Wonkwang University, Iksan, 570-749, Korea.

E-mail: yspkim@wonkwang.ac.kr

\section{Shoukat Ali}

Department of Mathematics, Govt. Engineering College Bikaner, Bikaner-334001, Rajasthan state, India.

E-mail: dr.alishoukat@rediffmail.com

Navratna Rathie

Department of Mathematics, Rajasthan Technical University,

Kota, Rajasthan State, India.

E-mail: navrathnarathie@yahoo.com 